\title{
Meta-analysis of the clinical and laboratory parameters of SFTS patients in China
}

Miao-miao Liu', Xiao-Ying Lei ${ }^{1}$ and Xue-jie $Y u^{1,2^{*}}$

\begin{abstract}
Background: Severe fever with thrombocytopenia syndrome (SFTS) is an emerging hemorrhagic fever in East Asia, which is caused by a novel bunyavirus-SFTSV. Many studies have reported the clinical characters of SFTS patients, but the reports were not consistent and a systematic summary of clinical manifestations and laboratory parameters are not available.

Method: A comprehensive literature research of Web of Science, PubMed, Wan Fang Data, and Chinese National Knowledge Infrastructure databases was conducted on articles which have described the clinical characters of SFTS patients. Data from selected studies were pooled by using STATA VERSION 12.0 software.

Result: Nine articles comprising 844 laboratory-confirmed SFTSV cases were included in this meta-analysis. The pooled case fatality rate was $16 \%$ (95\% Cl: 0.13-0.19). The major clinical characters of patients with SFTSV infection were fever, thrombocytopenia, leucopenia, gastrointestinal symptoms, and central nervous system manifestations. The risk factors for severe disease included bleeding tendency, central nervous system manifestations, elevated serum enzymes, and high viral load. Although there is no specific antiviral therapy for SFTSV infection, symptomatic treatment and supportive therapy including intensive monitoring is the most essential part of case management.

Conclusion: The major clinical characters of patients with SFTSV infection were fever, thrombocytopenia, leucopenia and gastrointestinal symptoms, and central nervous system manifestations. The risk factors for severity and fatality among SFTS patients included: old age, CNS manifestations, bleeding tendency, elevated serum enzymes, and high vial load.
\end{abstract}

Keywords: Severe fever with thrombocytopenia syndrome (SFTS), Case fatality rate, Clinical manifestation, Risk factor

\section{Key points}

Severe fever with thrombocytopenia syndrome is a severe hemorrhagic fever without effective treatment. Patients from endemic areas with fever, thrombocytopenia, leucopenia, gastrointestinal symptoms, and central nervous system manifestations should be considered as SFTS.

\section{Background}

Severe fever with thrombocytopenia syndrome (SFTS) is an emerging hemorrhagic fever, which was first discovered in rural areas of eastern and central China in 2009 [1] and more recently in South Korea and Japan [2, 3]. SFTS is caused by a novel bunyavirus-SFTS virus that

\footnotetext{
*Correspondence: xuyu@utmb.edu

${ }^{1}$ School of Public Health, Shandong University, 250012 Jinan, China

2Department of Pathology, University of Texas Medical Branch at Galveston, Galveston, TX 77555-0609, USA
}

has been detected from ticks and ticks are thought to be the vector of SFTSV [1, 4].

The major clinical symptoms of SFTS patients included acute fever (temperature of $38{ }^{\circ} \mathrm{C}$ or more), thrombocytopenia, leucopenia, gastrointestinal symptoms, and central nervous system (CNS) manifestations, followed with multiple organ dysfunctions [5-8]. Some cases in critical condition had the following manifestations: disturbance of consciousness, gastrointestinal bleeding, pulmonary hemorrhage, and skin bruising. The cases died due to diffuse intravascular coagulation (DIC), multiple organ failure, and shock [7-9].

Previous studies have confirmed that the outcome of the SFTS patients has been associated with the levels of their clinical characters and the biochemical markers at the early stage $[5-8,10]$. As the fatality rate of this disease was surprisingly high and the main risk factor for 
this phenomenon was not clear, this study was designed to analyze the relationship between various factors and the outcome of the SFTS patients through meta-analysis and to predict the severity of the disease.

\section{Methods}

\section{Search strategy}

We searched Web of Science, PubMed, Wan Fang Data, and Chinese National Knowledge Infrastructure databases from 2009 to 2016 using the following terms: ("SFTS" OR "SFTSV") and "patient". Moreover, we not only identified articles through database retrieval, but also by reviewing the reference list of retrieved articles to search for further relevant documents.

\section{Inclusion and exclusion criteria}

Articles included in this meta-analysis had to meet the following criteria: the first and foremost, the SFTS patient mentioned in the selected studies must be confirmed as meeting one or more of the following criteria: (1) isolated the virus from the patient's samples, (2) SFTSV RNA was detected from the patient's serum by a quantitative reverse-transcriptase polymerase chain reaction (qRT-PCR) and (3) a 4-fold or greater increase of antibody titers was detected between a paired serum samples of the patient collected from the acute and convalescent phases of infection; Secondly, the article contained the most recent or largest population was selected when the studies using the same or overlapping data by the same authors. Exclusion criteria included small scale studies with fewer than 15 patients, works designated as case reports, conference abstract, letters, editorials or reviews, and duplicated publications.

\section{Data extraction and quality assessment}

Based on the aforementioned inclusion and exclusion criteria, the preliminary screening was made by reading the title and abstract of the literature. Then, after reading the full text, the documents were eventually confirmed. All selected articles were independently screened and evaluated by two reviewers. After cross-checking, any disagreement was resolved by a third evaluator. The following information was extracted from every eligible article: the first author, year of publication, country, number of patients, number of fatalities, and the positive number of each clinical character.

\section{Statistical analysis}

I-squared was chosen to reflect the heterogeneity among studies. Values of $p<0.05$ and I-squared $>50 \%$ were considered to be statistically significant. According to the result of heterogeneity, the proper model was adopted to merge the clinical parameters of the patients: When the data were homogeneous, the fixed effect mode was used and for heterogeneous data, the random effect model was employed. Publication bias was assessed by using Egger's test: $p<0.05$ was considered to be statistically significant. For each selected publication, the pooled fatality rate, pooled positive rate of each clinical character, and their 95\% confidence intervals (CI) were calculated. We also calculated simple pooled rates along with a naive weighted average method [11]. All analyses were performed with STATA 12.0 (Stata Corp LP, College Station, Texas, United States).

\section{Results}

Literature search

A total of 241 relevant articles were retrieved after the preliminary screening from the electronic databases and other sources. Two hundred one of these literatures were excluded after review of the title and abstract for irrelevant topics, and an additional six documents were removed for duplication of the data. After reading the full text of the remaining 34 articles, 25 articles were excluded due to lack of some indicators. Nine studies were included for further meta-analysis. The data from these studies included in the meta-analysis was shown in Table 1.

\section{Basic information description}

A total of nine articles were included in this study. From the nine eligible studies, a total of 844 patients were included in this meta-analysis, which were published from 2011 to 2015. All the individuals were laboratoryconfirmed as SFTS patients. In all SFTS patients, the proportion of female was $48.1 \%$. The patients ranged from 7 to 89 years of age and most of them (80\%) were over 50 years old. Ninety-two percent of the patients were farmers living in wooded and hilly areas and working in the fields before onset of the disease. Most confirmed SFTS patients (96\%) involved in this analysis were admitted to the hospital from May to October.

\begin{tabular}{lllll}
\multicolumn{4}{l}{ Table 1 Summary of the studies included in the meta-analysis } \\
\hline Reference number & Author & Publication year & Country & Samples \\
\hline$[1]$ & Yu et al. & 2011 & China & 154 \\
{$[5]$} & Deng et al. & 2013 & China & 115 \\
{$[6]$} & Gai et al. & 2012 & China & 59 \\
{$[7]$} & Sun et al. & 2012 & China & 59 \\
{$[8]$} & Ding et al. & 2014 & China & 59 \\
{$[9]$} & Liu et al. & 2013 & China & 311 \\
{$[10]$} & Zhang et al. & 2012 & China & 49 \\
{$[32]$} & Cui et al. & 2013 & China & 16 \\
{$[33]$} & Wen et al. & 2014 & China & 22 \\
\hline
\end{tabular}


The case fatality rate and clinical character of SFTS patients The forest plot of case fatality rate of SFTS patients was shown in Fig. 1. The pooled case fatality rate was $16 \%$ (95\% CI: 0.13-0.19). By heterogeneity analysis, I-squared of case fatality rate was $10.6 \%(p=0.348)$, implying that there was no significant heterogeneity among the samples.

The pooled positive rates of clinical characters are shown in Table 2. As the definition of 'fever' was different in various studies, the heterogeneity of pooled positive rate of fever was $85.2 \%(p<0.05)$, it was recommended to choose the simple pooled rate to describe the situation of fever in SFTS patients. The simple pooled positive rate of fever was $94 \%$. As the I-squared of pooled rate of thrombocytopenia was $71 \%$ ( $p=0.03$ ), we chose the simple pooled rate to reflect the combined effects. Symptomatic patients comprised $65 \%$ of the group. All the studies have defined 'leucopenia' as leukocyte count $<4.0 \times 10^{9} / \mathrm{L}$, and $88 \%$ of patients presented with this laboratory finding. Patients with SFTSV infection usually have gastrointestinal symptoms, including anorexia, nausea, vomiting, diarrhea or abdominal pain. The polled positive rate of those symptoms were $83 \%, 54 \%, 39 \%, 34 \%, 22 \%$, respectively. In addition, patients with SFTSV infection also had hemorrhagic manifestations and central nervous system manifestations (Table 2).

\section{Laboratory parameters of SFTS patients}

During the early stage of SFTSV infection, the serum viral load was high both in non-fatal and fatal patients.
Nevertheless, along with the development of the disease, the serum viral load decreased in non-fatal patients, but still remained at a high level in fatal cases. All the selected literatures showed that SFTSV infection was associated with liver and kidney function impairment. Laboratory examination found an increase of serum enzymes, such as aspartate aminotransferase (AST), alanine aminotransferase (ALT), lactate dehydrogenase (LDH), creatine kinase (CK), as well as creatine kinase $\mathrm{MB}$ fraction (CK-MB), which indicated the liver dysfunction. The level of serum enzymes increased to a peak about 10 days after onset in non-fatal SFTS cases, and then gradually declined to normal level, however, it progressively increased in fatal cases. Similar to the above findings, blood urea nitrogen (BUN) also increased in SFTS patients, which is a parameter that can indicate impairment of renal function. Furthermore, in all detected clinical laboratory parameters, blood coagulation times (activated partial thromboplastin time, APTT and thrombin time, TT) were longer among SFTS patients than the healthy individuals. In addition, cytokine levels, such as interleukin-6 (IL-6), IL-8, IL-10, granulocyte colony-stimulating factor (G-CSF), and interferon- $\gamma$ (IFN- $\gamma$ ) were enhanced in SFTS patients and were significantly higher in fatal cases than the non-fatal individuals.

\section{Discussion}

Most cases are farmers (92\%), who were living or working in wooded and hill areas, where ticks were commonly found. This phenomenon indicated that ticks are most

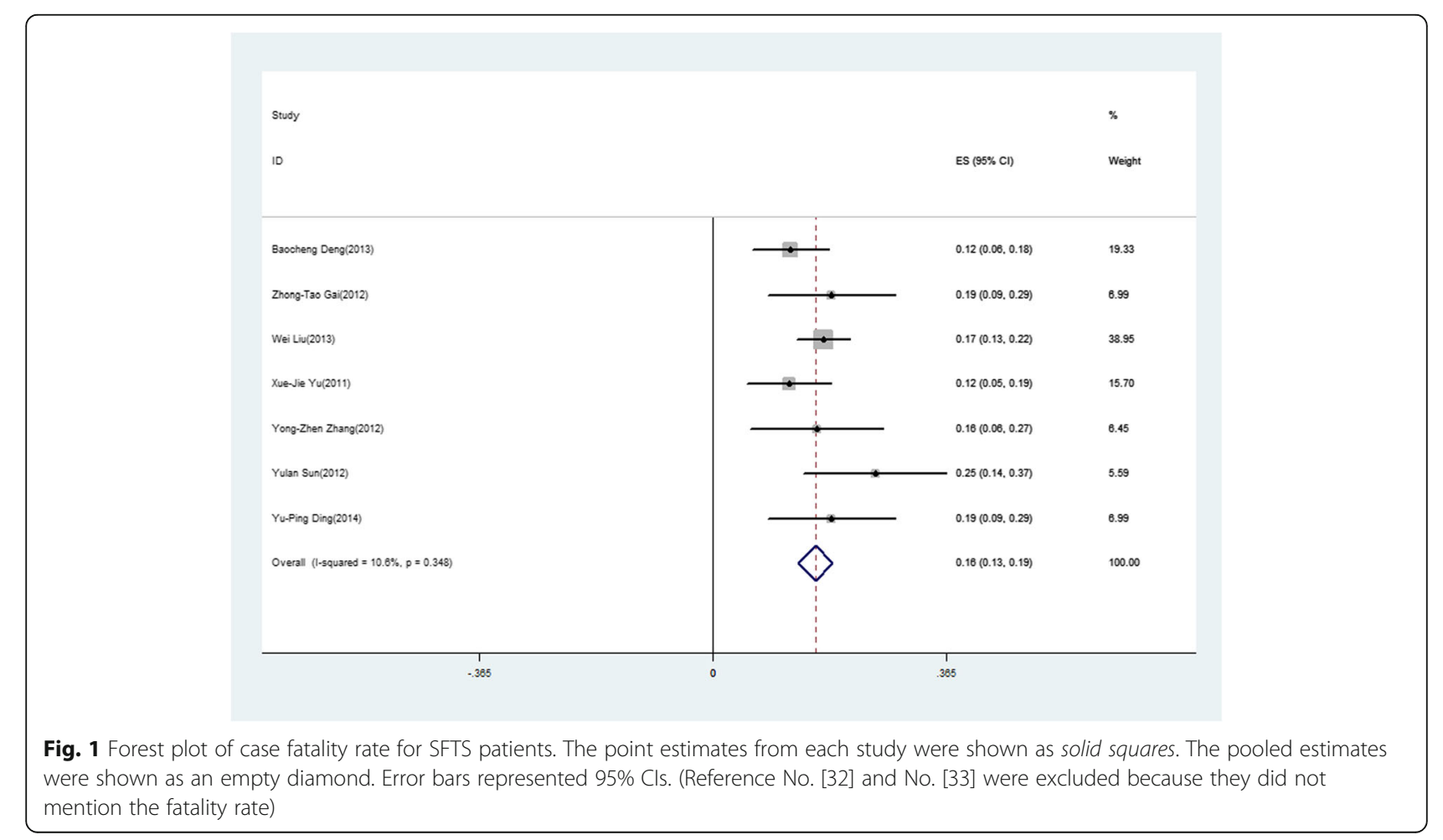


Table 2 The pooled positive rates of clinical characters ${ }^{a}$

\begin{tabular}{|c|c|c|c|c|c|c|}
\hline & & & Pooled & $95 \%$ & & Simple pooled \\
\hline & $I^{2}(\%)$ & $p$ & Rate & Low & Up & Rate \\
\hline Fever $^{b}$ & 85.20 & $<0.05$ & 0.99 & 0.97 & 1.00 & 0.94 \\
\hline Leucopenia $^{c}$ & 0.00 & 0.70 & 0.88 & 0.82 & 0.94 & 0.73 \\
\hline Thrombocytopenia ${ }^{d}$ & 71.00 & 0.03 & 0.83 & 0.51 & 1.16 & 0.65 \\
\hline Anorexia & 58.70 & 0.09 & 0.83 & 0.76 & 0.91 & 0.85 \\
\hline Weakness & 46.70 & 0.09 & 0.74 & 0.54 & 0.94 & 0.83 \\
\hline Nausea & 34.60 & 0.21 & 0.54 & 0.49 & 0.60 & 0.55 \\
\hline Myalgias & 49.60 & 0.09 & 0.51 & 0.42 & 0.60 & 0.62 \\
\hline Proteinuria & 62.30 & 0.10 & 0.50 & 0.39 & 0.61 & 0.51 \\
\hline Lymphadenopathy & 61.80 & 0.05 & 0.39 & 0.29 & 0.49 & 0.35 \\
\hline Vomiting & 55.50 & 0.05 & 0.39 & 0.33 & 0.46 & 0.38 \\
\hline Joint pain & 0.00 & 0.33 & 0.35 & 0.27 & 0.43 & 0.28 \\
\hline Chills & 30.10 & 0.24 & 0.32 & 0.22 & 0.43 & 0.27 \\
\hline Diarrhea & 66.60 & 0.02 & 0.31 & 0.23 & 0.39 & 0.34 \\
\hline Hematuria & 25.10 & 0.25 & 0.29 & 0.22 & 0.36 & 0.29 \\
\hline Cough & 67.60 & 0.08 & 0.28 & 0.20 & 0.36 & 0.26 \\
\hline Abdominal pain & 26.80 & 0.25 & 0.22 & 0.15 & 0.29 & 0.29 \\
\hline Dizziness & 0.00 & 0.53 & 0.20 & 0.16 & 0.24 & 0.25 \\
\hline Headache & 19.80 & 0.29 & 0.17 & 0.13 & 0.21 & 0.22 \\
\hline Case fatality rate & 10.60 & 0.35 & 0.16 & 0.13 & 0.19 & 0.16 \\
\hline Petechiae & 16.70 & 0.31 & 0.08 & 0.06 & 0.11 & 0.11 \\
\hline
\end{tabular}

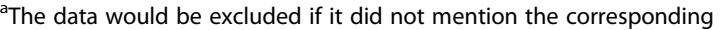
clinical character

${ }^{b}$ Fever was defined as body temperature $\geq 38^{\circ} \mathrm{C}$ in references $[1,5,32,33]$; $\geq 37.5^{\circ} \mathrm{C}$ in references $[8,9] ; \geq 39{ }^{\circ} \mathrm{C}$ in reference [6]

CLeucopenia was defined as leukocyte count $<4.0 \times 10^{9} / \mathrm{L}$

${ }^{\mathrm{d}}$ Thrombocytopenia was defined as platelet count $<150 \times 10^{9} / \mathrm{L}$ in references $[5,32,33] ;<100 \times 10^{9} / L$ in references $[1,8-10]$

likely to be the vector of SFTSV $[1,4,12]$. Most patients were admitted to hospital from May to October. During this period, farmers were engaged in the agricultural activities, and were easily exposed to ticks. This interpretation indirectly confirmed that ticks are the vector of SFTSV. In addition, it has been confirmed that SFTSV can be transmitted to humans by contact with blood or body fluid from SFTS patients [13, 14]. Therefore, individuals should not only pay attention to reduce exposure to ticks in daily life, but also pay attention to personal protection when they take care of SFTS patients.

The disease mainly occurs in the elderly population $[15,16]$. Consistent with the aforementioned results, in this study, the vast majority of the patients are over 50 years old. Studies revealed that older age was a significant risk factor for fatal outcome of SFTS patients $[8,9,17,18]$. The cause of this phenomenon-older patients tended to have more severe symptoms, is likely to be that the older people have lower immunological capacity to limit the replication of SFTSV. The high incidence of SFTS in old people could be caused by a population skew toward to old people in countryside because many young people left rural area to work in city. However, a previous study indicated the SFTSV antibody positive rate was not significantly different among people at different age groups in rural area, suggesting that age is the critical risk factor to determine for SFTS morbidity and mortality [19].

The typical features of SFTS are acute fever and thrombocytopenia. About $94 \%$ of patients had acute fever at the early stage and most of them returned to normal in 10 days. At the early stage of infection, as exogenous stimuli, the virus activated the body to release endogenous pyrogens. Endogenous pyrogens, in turn, circulate to the thermoregulatory center of the brain where it cause an elevation of the body temperature [20]. Over $65 \%$ of patients had a lower platelet count (platelet count $<150 \times 10^{9} / \mathrm{L}$ ). Both in vitro and in vivo assays confirmed that the virus adhered to platelets and facilitated the phagocytosis of platelets by macrophages $[8,21]$. Those patients with thrombocytopenia often accompanied with hemorrhagic tendency, such as petechiae and microscopic hematuria. In addition, severe hemorrhagic symptoms, such as gastrointestinal bleeding, macroscopic hematuria and hematoma, are commonly observed in sever patients with disseminated intravascular coagulation [3]. In comparison with SFTS patients who survived, the deceased patients had a more serious hemorrhagic tendency. It suggests that bleeding tendency was associated with the death of SFTS patients.

The central nervous system symptoms commonly occurred approximately 5 days after the onset of illness, and persisted for 1-2 weeks [6]. The CNS manifestations of SFTS patients included dizziness, headache, apathy and coma before death. The CNS manifestations of deceased patients were more serious than survivors indicated that CNS manifestations are the risk factors for the death of SFTS patients.

In SFTS patients' serum, the levels of liver, kidney, and cardiac enzymes are commonly dramatic elevated, indicating the impairments of those organs. In survivors, the serum enzymes reached to the maximum levels in about 10 days after infection, and then gradually declined to the normal levels. However, in deceased patients, the levels of serum enzymes appeared to progressively rise and reached to the maximal values before death. The risk analysis revealed that elevated AST, LDH, CK and CK-MB were risk factors associated with severity among SFTS patients and fatality among severe SFTS patients $[5,6]$.

Levels of serum cytokines such as IL-6, IL-10, G-CSF, and IFN- $\gamma$ were increased in SFTSV-infected patients. Clinical studies have indicated that cytokine storm, characterized by overproduction of certain cytokines, is commonly associated with the severity of the disease $[5,7]$. Consistent with the findings aforementioned, the virus 
infection including hemorrhagic fever virus, such as, Crimean Congo Hemorrhagic Fever (CCHF), Ebola, and Rift Valley fever virus can lead to cytokine storm which is associated with the progression of the disease [22-24]. Cytokine storm occurring in the acute phase of the disease has been widely hypothesized to be the main cause of morbidity and mortality for SFTSV infection [25]. Under normal circumstances, inflammatory cytokine and chemokine could promote activation and chemoattraction of lymphocytes and exert antiviral effects. However, recent findings indicated that cytokine storm, characterized by an overwhelming and imbalanced profile of cytokines, could become excessive and harmful [26].

Serum viral load was considered to be the major laboratory marker for clinical diagnosis. After the onset of the illness, the initial serum viral load was high in all patients. Then the virus was gradually cleared in survivors at the recovery phase, but still remained high in the fatal patients. There was a difference between the serum viral load of the surviving and deceased patients [8, 27-29]. High serum viral load was considered to be the high risk factor that resulted in the death of SFTS patients [10]. Therefore, it was identified as an important parameter to predict the outcome of the SFTS patients. Meanwhile, studies founded that there was a positive correlation between the serum viral load and the levels of various cytokines. In other words, during the acute phase of SFTSV infection, the higher viral load, the higher levels of cytokines (except for RANTES and PDGF-BB) [7, 28]. These findings suggested that the high viral load may lead to excessive secretion of cytokines, which could further aggravate the progression of the disease.

Ribavirin is reported to be effective for treating CCHF infections and hemorrhagic fever with renal syndrome $[30,31]$. However, studies have found that intravenous ribavirin had no effect on reducing the serum viral load in SFTS patients [5, 9]. Meanwhile, the use of tetracycline for the treatment in SFTS patients is not justified unless co-infection can be confirmed [32]. In addition, treatment of SFTS patients with corticosteroids for acute respiratory distress syndrome is controversial. This is because though corticosteroids can suppress the cytokine storm, it also can increase the risk of developing critical disease from viral infection [5]. Hence, supportive therapy is the most essential and effective part of case management [33].

Our meta-analysis has some limitations: the primary limitation of our meta-analysis was that there are subtle differences in the definition of the clinical characters in different studies. Since in different articles, the forms of the laboratory parameters were not uniform, we could not carry out a systematic analysis for these indicators. Also, like most other meta-analysis, the overall findings from the meta-analysis were limited by the quality of the primary studies.

\section{Conclusion}

In conclusion, the major clinical characters of patients with SFTSV infection were fever, thrombocytopenia, leucopenia and gastrointestinal symptoms, and central nervous system manifestations. The risk factors for severity and fatality among SFTS patients included: old age, CNS manifestations, bleeding tendency, elevated serum enzymes, and high vial load.

\section{Funding}

This study was supported by grants from Shandong Province Science and Technology Development Program (no. 2014GSF121004), Natural Science Foundation of Shandong Province, China (ZR2014HP025), National Natural Science Funds of China (31570167; 81401368).

\section{Authors' contribution}

MML wrote the manuscript. All authors reviewed the manuscript. All authors read and approved the final manuscript.

\section{Competing interest}

The authors declare that they have no competing interests.

\section{Ethics approval and consent to participate}

This study was carried out in accordance with the Guidelines of Regulations for the Administration of Laboratory Animals (Decree No. 2 of the State Science and Technology Commission of the People's Republic of China, 1988). The experimental research reported in this study was approved by the bioethics committee of School of Public Health, Shandong University. Human research was carried out in compliance with the Declaration of Helsinki.

Received: 1 September 2016 Accepted: 25 November 2016 Published online: 29 November 2016

\section{References}

1. Yu XJ, Liang MF, Zhang SY, Liu Y, Li JD, Sun YL, Zhang L, Zhang QF, Popov $V L$, Li C, et al. Fever with thrombocytopenia associated with a novel bunyavirus in China. N Engl J Med. 2011;364(16):1523-32.

2. Kim KH, Yi J, Kim G, Choi SJ, Jun Kl, Kim NH, Choe PG, Kim NJ, Lee JK, Oh MD. Severe fever with thrombocytopenia syndrome, South Korea, 2012. Emerg Infect Dis. 2013;19(11):1892-4.

3. Takahashi T, Maeda K, Suzuki T, Ishido A, Shigeoka T, Tominaga T, Kamei T, Honda M, Ninomiya D, Sakai T, et al. The first identification and retrospective study of Severe Fever with Thrombocytopenia Syndrome in Japan. J Infect Dis. 2014;209(6):816-27.

4. Luo LM, Zhao L, Wen HL, Zhang ZT, Liu JW, Fang LZ, Xue ZF, Ma DQ, Zhang XS, Ding SJ, et al. Haemaphysalis longicornis Ticks as Reservoir and Vector of Severe Fever with Thrombocytopenia Syndrome Virus in China. Emerg Infect Dis. 2015;21(10):1770-6.

5. Deng B, Zhou B, Zhang S, Zhu Y, Han L, Geng Y, Jin Z, Liu H, Wang D, Zhao $Y$, et al. Clinical features and factors associated with severity and fatality among patients with severe fever with thrombocytopenia syndrome Bunyavirus infection in Northeast China. PLoS One. 2013;8(11):e80802.

6. Gai ZT, Zhang Y, Liang MF, Jin C, Zhang S, Zhu CB, Li C, Li XY, Zhang QF, Bian PF, et al. Clinical progress and risk factors for death in severe fever with thrombocytopenia syndrome patients. J Infect Dis. 2012;206(7):1095-102.

7. Sun Y, Jin C, Zhan F, Wang X, Liang M, Zhang Q, Ding S, Guan X, Huo X, Li $C_{\text {, et }}$ al. Host cytokine storm is associated with disease severity of severe fever with thrombocytopenia syndrome. J Infect Dis. 2012;206(7):1085-94.

8. Ding YP, Liang MF, Ye JB, Liu QH, Xiong CH, Long B, Lin WB, Cui N, Zou ZQ, Song $Y L$, et al. Prognostic value of clinical and immunological markers in acute phase of SFTS virus infection. Clin Microbiol Infect. 2014;20(11):0870-8.

9. Liu W, Lu QB, Cui N, Li H, Wang LY, Liu K, Yang ZD, Wang BJ, Wang HY, Zhang $Y Y$, et al. Case-fatality ratio and effectiveness of ribavirin therapy among hospitalized patients in china who had severe fever with thrombocytopenia syndrome. Clin Infect Dis. 2013;57(9):1292-9.

10. Zhang YZ, He YW, Dai YA, Xiong Y, Zheng H, Zhou DJ, Li J, Sun Q, Luo XL, Cheng $Y L$, et al. Hemorrhagic fever caused by a novel Bunyavirus in China: pathogenesis and correlates of fatal outcome. Clin Infect Dis. 2012;54(4):527-33. 
11. Ali SA, Donahue RM, Qureshi H, Vermund SH. Hepatitis B and hepatitis C in Pakistan: prevalence and risk factors. Int J Infect Dis. 2009;13(1):9-19.

12. Ding S, Yin H, Xu X, Liu G, Jiang S, Wang W, Han X, Liu J, Niu G, Zhang X, et al. A cross-sectional survey of severe fever with thrombocytopenia syndrome virus infection of domestic animals in Laizhou City, Shandong Province, China. Jpn J Infect Dis. 2014;67(1):1-4.

13. Bao CJ, Guo XL, Qi X, Hu JL, Zhou MH, Varma JK, Cui LB, Yang HT, Jiao YJ, Klena JD, et al. A family cluster of infections by a newly recognized bunyavirus in eastern China, 2007: further evidence of person-to-person transmission. Clin Infect Dis. 2011;53(12):1208-14.

14. Liu Y, Li Q, Hu W, Wu J, Wang Y, Mei L, Walker DH, Ren J, Wang Y, Yu XJ. Person-to-person transmission of severe fever with thrombocytopenia syndrome virus. Vector Borne Zoonotic Dis. 2012;12(2):156-60.

15. Liu K, Zhou H, Sun RX, Yao HW, Li Y, Wang LP, Mu D, Li XL, Yang Y, Gray GC, et al. A national assessment of the epidemiology of severe fever with thrombocytopenia syndrome, China. Sci Rep. 2015;5:9679.

16. Zhang X, Liu Y, Zhao L, Li B, Yu H, Wen H, Yu XJ. An emerging hemorrhagic fever in China caused by a novel bunyavirus SFTSV. Sci China Life Sci. 2013;56(8):697-700

17. Weng Y, Chen N, Han Y, Xing Y, Li J. Clinical and laboratory characteristics of severe fever with thrombocytopenia syndrome in Chinese patients. Braz J Infect Dis. 2014;18(1):88-91.

18. Sun J, Chai C, Lv H, Lin J, Wang C, Chen E, Zhang Y, Chen Z, Liu S, Gong Z, et al. Epidemiological characteristics of severe fever with thrombocytopenia syndrome in Zhejiang Province, China. Int J Infect Dis. 2014;25:180-5.

19. Ding S, Niu G, Xu X, Li J, Zhang X, Yin H, Zhang N, Jiang X, Wang S, Liang $M$, et al. Age is a critical risk factor for severe fever with thrombocytopenia syndrome. PLoS One. 2014;9(11):e111736.

20. Bernheim HA, Block LH, Atkins E. Fever: pathogenesis, pathophysiology, and purpose. Ann Intern Med. 1979;91(2):261-70.

21. Jin C, Liang M, Ning J, Gu W, Jiang H, Wu W, Zhang F, Li C, Zhang Q, Zhu H, et al. Pathogenesis of emerging severe fever with thrombocytopenia syndrome virus in C57/BL6 mouse model. Proc Natl Acad Sci U S A. 2012;109(25):10053-8.

22. Papa A, Dalla V, Papadimitriou E, Kartalis GN, Antoniadis A. Emergence of Crimean-Congo haemorrhagic fever in Greece. Clin Microbiol Infect. 2010;16(7):843-7.

23. Hutchinson KL, Rollin PE. Cytokine and chemokine expression in humans infected with Sudan Ebola virus. J Infect Dis. 2007;196 Suppl 2:S357-63.

24. McElroy AK, Nichol ST. Rift Valley fever virus inhibits a pro-inflammatory response in experimentally infected human monocyte derived macrophages and a pro-inflammatory cytokine response may be associated with patient survival during natural infection. Virology. 2012;422(1):6-12.

25. Chensue SW. Molecular machinations: chemokine signals in host-pathogen interactions. Clin Microbiol Rev. 2001;14(4):821-35. table of contents.

26. Wang $\mathrm{H}, \mathrm{Ma} \mathrm{S}$. The cytokine storm and factors determining the sequence and severity of organ dysfunction in multiple organ dysfunction syndrome. Am J Emerg Med. 2008;26(6):711-5.

27. Li J, Han Y, Xing Y, Li S, Kong L, Zhang Y, Zhang L, Liu N, Wang Q, Wang S, et al. Concurrent measurement of dynamic changes in viral load, serum enzymes, T cell subsets, and cytokines in patients with severe fever with thrombocytopenia syndrome. PLoS One. 2014;9(3):e91679.

28. Li D. A highly pathogenic new bunyavirus emerged in China. Emerg Microbes Infect. 2013;2(1):e1.

29. Liu Q, He B, Huang S-Y, Wei F, Zhu X-Q. Severe fever with thrombocytopenia syndrome, an emerging tick-borne zoonosis. Lancet Infect Dis. 2014;14(8):763-72.

30. Ergonul O, Celikbas A, Dokuzoguz B, Eren S, Baykam N, Esener H. Characteristics of patients with Crimean-Congo hemorrhagic fever in a recent outbreak in Turkey and impact of oral ribavirin therapy. Clin Infect Dis. 2004:39(2):284-7.

31. Huggins JW, Hsiang CM, Cosgriff TM, Guang MY, Smith Jl, Wu ZO, LeDuc JW, Zheng ZM, Meegan JM, Wang QN, et al. Prospective, double-blind, concurrent, placebo-controlled clinical trial of intravenous ribavirin therapy of hemorrhagic fever with renal syndrome. J Infect Dis. 1991;164(6):1119-27.

32. Cui F, Cao HX, Wang L, Zhang SF, Ding SJ, Yu XJ, Yu H. Clinical and epidemiological study on severe fever with thrombocytopenia syndrome in Yiyuan County, Shandong Province, China. Am J Trop Med Hyg. 2013;88(3):510-2.

33. Wen HL, Zhao L, Zhai S, Chi Y, Cui F, Wang D, Wang L, Wang Z, Wang Q, Zhang $\mathrm{S}$, et al. Severe fever with thrombocytopenia syndrome, Shandong Province, China, 2011. Emerg Infect Dis. 2014;20(1):1-5.

\section{Submit your next manuscript to BioMed Central and we will help you at every step:}

- We accept pre-submission inquiries

- Our selector tool helps you to find the most relevant journal

- We provide round the clock customer support

- Convenient online submission

- Thorough peer review

- Inclusion in PubMed and all major indexing services

- Maximum visibility for your research

Submit your manuscript at www.biomedcentral.com/submit
C) Biomed Central 